\title{
Spontaneous alternation behavior in humans
}

\author{
Nguyen, Anh Thy H ; Kunz, Andreas ; Rothacher, Yannick ; Brugger, Peter ; Lenggenhager, Bigna
}

\begin{abstract}
Redirected walking refers to a number of techniques that enable users to explore a virtual environment larger than the physical space by real walking. The efficiency of these techniques has been shown to improve when predictions about the user's future trajectory are incorporated. Predictions can be made not only based on the knowledge about the environment but also on how humans behave in it. In a maze-like environment, it is known that most animal species show a strong preference to alternate their turning direction. This is called spontaneous alternation behavior (SAB). Although such behavior has also been observed in humans during maze tracing tasks, little is known whether they also exhibit this behavior during real walking, and if they do, what their alternation rate is. In the experiment described in this paper, 60 right-handed subjects were invited to walk freely through a virtual maze consisting of a primary $90^{\circ}$ forced turn followed by three consecutive T-junctions. Results show that, on average, humans exhibited an alternation rate of $72 \%$. When looking only at the junction after the forced turn, subjects alternated with $76 \%$. After two consecutive turns of the same direction subjects alternated with $93 \%$. The alternation rates obtained not only clearly confirm the existence of SAB in humans but also could be used to improve the accuracy of existing prediction models in human walking.
\end{abstract}

DOI: https://doi.org/10.1145/3139131.3139168

Posted at the Zurich Open Repository and Archive, University of Zurich

ZORA URL: https://doi.org/10.5167/uzh-212283

Conference or Workshop Item

Published Version

The following work is licensed under a Publisher License.

Originally published at:

Nguyen, Anh Thy H; Kunz, Andreas; Rothacher, Yannick; Brugger, Peter; Lenggenhager, Bigna (2017). Spontaneous alternation behavior in humans. In: VRST '17: 23rd ACM Symposium on Virtual Reality Software and Technology, Gothenburg, 8 November 2017 - 10 November 2017. ACM, 1-4.

DOI: https://doi.org/10.1145/3139131.3139168 


\section{Spontaneous Alternation Behavior in Humans}

\author{
Anh Nguyen \\ Andreas Kunz \\ nngoc@ethz.ch \\ kunz@iwf.mavt.ethz.ch \\ Innovation Center Virtual Reality, ETH Zurich \\ Leonhardstrasse 21, LEE L201 \\ Zurich 8092, Switzerland
}

\author{
Yannick Rothacher \\ Peter Brugger \\ Bigna Lenggenhager \\ Yannick.Rothacher@usz.ch \\ Peter.Brugger@usz.ch \\ bigna.lenggenhager@gmail.com \\ Neuropsychology, University Hospital Zurich \\ Sonneggstrasse 6 \\ Zurich 8006, Switzerland
}

\begin{abstract}
Redirected walking refers to a number of techniques that enable users to explore a virtual environment larger than the physical space by real walking. The efficiency of these techniques has been shown to improve when predictions about the user's future trajectory are incorporated. Predictions can be made not only based on the knowledge about the environment but also on how humans behave in it. In a maze-like environment, it is known that most animal species show a strong preference to alternate their turning direction. This is called spontaneous alternation behavior (SAB). Although such behavior has also been observed in humans during maze tracing tasks, little is known whether they also exhibit this behavior during real walking, and if they do, what their alternation rate is.

In the experiment described in this paper, 60 right-handed subjects were invited to walk freely through a virtual maze consisting of a primary $90^{\circ}$ forced turn followed by three consecutive T-junctions. Results show that, on average, humans exhibited an alternation rate of $72 \%$. When looking only at the junction after the forced turn, subjects alternated with $76 \%$. After two consecutive turns of the same direction subjects alternated with $93 \%$. The alternation rates obtained not only clearly confirm the existence of SAB in humans but also could be used to improve the accuracy of existing prediction models in human walking.
\end{abstract}

\section{CCS CONCEPTS}

- Human-centered computing $\rightarrow$ Virtual reality; • Applied computing $\rightarrow$ Psychology;

\section{KEYWORDS}

Spontaneous Alternation Behaviour (SAB), Redirected Walking, Model Predictive Control

Permission to make digital or hard copies of all or part of this work for personal or classroom use is granted without fee provided that copies are not made or distributed for profit or commercial advantage and that copies bear this notice and the full citation on the first page. Copyrights for components of this work owned by others than ACM must be honored. Abstracting with credit is permitted. To copy otherwise, or republish, to post on servers or to redistribute to lists, requires prior specific permission and/or a fee. Request permissions from permissions@acm.org.

VRST '17, November 8-10, 2017, Gothenburg, Sweden

(C) 2017 Association for Computing Machinery.

ACM ISBN 978-1-4503-5548-3/17/11 ..\$15.00

https://doi.org/10.1145/3139131.3139168

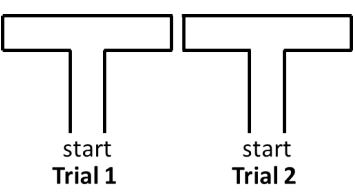

(a) T-maze

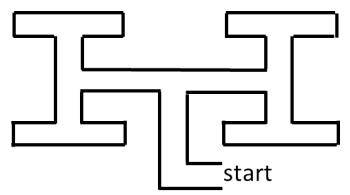

(b) Forced maze
Figure 1: Types of mazes

ACM Reference format:

Anh Nguyen, Andreas Kunz, Yannick Rothacher, Peter Brugger, and Bigna Lenggenhager. 2017. Spontaneous Alternation Behavior in Humans. In Proceedings of VRST '17, Gothenburg, Sweden, November 8-10, 2017, 4 pages. https://doi.org/10.1145/3139131.3139168

\section{INTRODUCTION}

Spontaneous alternation behavior (SAB) refers to an animal's tendency to avoid consecutive repeated choices when exploring a maze. In a classical $\mathrm{SAB}$ test, an animal is placed at the start stem of a T-maze (Figure 1a) at the beginning of two trials. In each trial, the animal moves through the start stem and enters one goal arm. If in the first trial the animal enters one goal arm, in the second one it will tend to choose the other arm, and thus "alternate", with above chance probability. Although there is numerous research on such behavior in various animal species, not much has been done on humans, particularly adults. Until 1980, most SAB studies in humans involved children tracing mazes using pen and paper [Harris 1971], or sequentially pressing two buttons to turn on lights of different colors [Miller et al. 1969]. In [Pate and Bell 1971], preschool children from 3 to 6 years old were asked to crawl across a T-maze tunnel two times, and the average alternation rate was found to be $56.4 \%$, which did not differ from chance. With regards to studies in human adults, in [Lawless and Engstrand 1960], university students were asked to use a stylus to trace a forced maze (Figure 1b) blindfolded and results showed that the alternation rate is $75 \%$. The only literature found where SAB was tested on walking adults is [Neiberg et al. 1970], a study in which college students were asked to walk through a "T-corridor" two times. Their alternation rate was shown to lie at $60 \%$. It is, however, unclear what the exact instructions to the subjects were, and whether they might have affected the spontaneity of their behavior. 
At this point, question may arise why we choose to investigate $\mathrm{SAB}$ in humans after years of interrupted research. The answer to this question is twofold. The first reason lies in the development and increasing availability of virtual reality (VR) technology. While it must have been complicated in the 80 s to build an apparatus suited for testing SAB in human walking, VR technology opens the door to countless possibilities to build and design various virtual environments at no significant incremental cost. Moreover, the combination of tracking technology and VR allows users to walk naturally in a virtual environment instead of having to stay in place while tracing mazes using a stylus. In order to elaborate on the second reason to study SAB in humans, we would like first to introduce the concepts of redirected walking (RDW) and RDW planners using model predictive control (MPC). RDW refers to techniques that enable the users to explore a virtual environment (VE) larger than the physical space. First introduced in [Razzaque et al. 2001], the RDW techniques involve "manipulating" the mapping between the virtual and real space. These techniques are applied on different aspects of walking: translation - the users walk slower/faster in the VE than in real life, rotation - the users rotate slower or faster in the VE than in real life, and curvature - the users walk on a curve in real life while still going straight in the VE. When applied within certain thresholds, these manipulations will go unnoticed and immersion can be maintained [Grechkin et al. 2016] [Steinicke et al. 2008]. In case of limited physical space, these RDW techniques could be used in different manners to steer users away from the physical boundary, and when these methods fail and collisions still occur, "resets" could be applied to reorient users towards open space [Williams et al. 2007]. For example, the steer-to-center, steer-to-orbit, and steer-to-changing-targets algorithms use RDW techniques to steer users toward the center, a circular path around the center, and towards one of the predefined points in the physical space respectively [Razzaque et al. 2001]. On the other hand, the MPC algorithm chooses where users should be directed to, a socalled RDW action, based on the prediction of their next $\mathrm{N}$ moves [Nescher et al. 2014]. This RDW action is chosen to minimize a cost function which penalizes the use of resets, therefore the number of resets is also minimized. In [Nescher et al. 2014], the MPC algorithm was shown to reduce the number of resets by $41 \%$ compared to the steer-to-center algorithm in a maze-like environment. The efficiency improvement could be accredited to the usage of users' movement prediction in the planning process. This prediction is expressed as a probability distribution and so far is obtained based on relatively basic assumptions such as: under normal circumstances, users don't walk through virtual obstacles or walls; in a T-maze condition, the probability of turning right or left is $50 \%$; etc. If more knowledge about how users behave is obtained, prediction's accuracy can be improved, consequently enhancing the efficiency of the MPC algorithm.

From this angle, we would like to design an experiment to investigate whether humans really exhibit $S A B$ behavior, and what their alternation rate is if they do. The results of this experiment not only allow us better understanding of how humans behave, but also contribute to the further improvement of existing RDW technology.

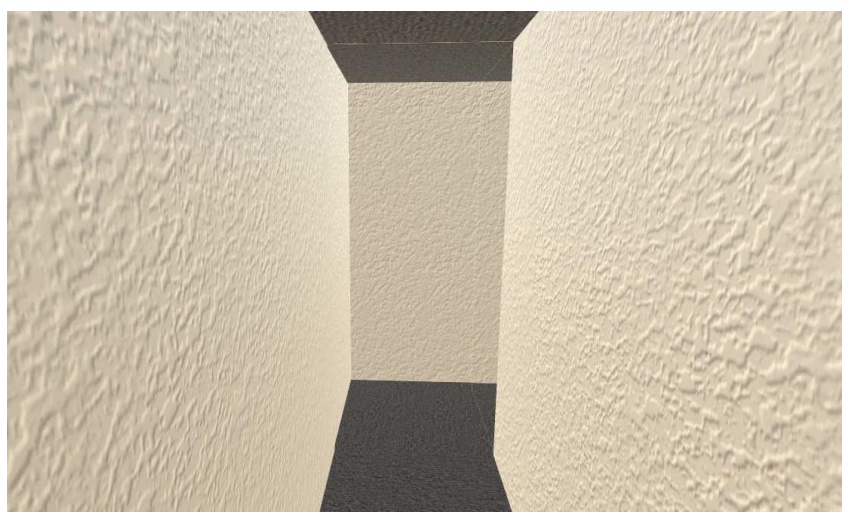

Figure 2: Maze view from the starting position, before the forced turn

\section{USER STUDY}

\subsection{Experiment Design}

In the classical T-maze (Figure 1a), SAB is quantified as the alternation rate, namely the percent of times the subjects alternate turn directions. In an extended forced maze (Figure 1b), where subjects perform a number of forced turns before the free turns at the Tjunctions (Figure 1b), SAB could be quantified as the conditional probability of alternation given the direction of the previous turn(s). While both designs are suitable for investigating the existence of $\mathrm{SAB}$, we selected the extended force maze over the classical T-maze as the environment is more similar to a natural setting. Moreover, an extended forced maze also offers the possibility to observe whether users maintain or change their alternation rate as they walk further into the maze.

The final maze design consists of an initial $90^{\circ}$ forced turn, which is followed by three consecutive T-junctions (Figure 1b). Unit pieces of the maze such as straight, corner and T-joint are first modeled in Blender and then imported and assembled in Unity. The maze walls have a generic plaster texture (Figure 2), and lights are placed in the environment such that when the subjects are at the T-intersections, both choices look completely identical. The distance between each turn is $1.5 \mathrm{~m}$. Due to limited lab space, the complete maze can not all fit in. As a result, only parts of the environment are shown at a time depending on the position of the subjects This approach is similar to the flexible spaces approach introduced in [Vasylevska et al. 2013]. Step regulating sound is played during the subjects' walk in the maze to ensure a walking speed of $0.75 \mathrm{~m} / \mathrm{s}$ for all subjects. The height information of subjects was collected before the experiment day, and the step sound prepared in advance using the formula in [Dean 1965]. This fixed walking speed is used to ensure subjects walk continuously through the maze without stopping.

\subsection{Experiment Setup}

The experiment setup consists of an Oculus DK2 and an Intersense IS-1200 optical tracking system mounted on top, providing 6 DOF positional tracking at $180 \mathrm{~Hz}$. The scene is optimally designed in Unity to run at the HMD's maximum frame rate $75 \mathrm{~Hz}$. The whole setup is powered by a backpack-mounted notebook. The available tracking space is $12 \mathrm{~m} \times 6 \mathrm{~m}$. 


\subsection{Participants and procedure}

60 subjects (aged from 18-35 (mean=25.2, $\mathrm{SD}=3.8)$, 30 male and 30 female, right-handed, with normal or corrected-to-normal vision) were recruited through the university market place. The SAB experiment lasted about 15 minutes including instructions and set up time. The subjects were not informed about the purpose of the experiment. The direction of the initial forced turn was balanced across subjects. Before starting the experiment subjects were shown screen shots of different parts of the experiment and were given this instruction: "When the program starts, you will see a starting position. Walk to this position. A maze will appear (Figure 2) and you will hear ongoing step sounds. Please follow this step rhythm while walking through the maze without stopping. You can freely explore the maze as you want." After signing the consent form, subjects put on the backpack and started walking. An experimenter walked right behind the subjects to prevent them from colliding with the physical walls in the event that they would not follow the path of the VR maze. After the subjects completed the experiment, they were asked: "Did you use any strategy or plan where to go? Or were you just walking spontaneously?" Their answer to this question determined whether their data would be used for the analysis. Subjects were paid $15 \mathrm{CHF} /$ hour for their contribution.

\section{RESULTS}

When asked if they used any strategy for the maze, out of 60 subjects, 11 subjects ( 8 male and 3 female) answered similar to one of the following ways: "I was trying to see if I can walk in a circle", "I deliberately changed directions so that I can walk further", or "I decided to always turn left". The data from these subjects was not included in the analysis. The remaining 49 subjects answered that they were walking spontaneously without any planning.

The three consecutive direction choices per subject were scored either as alternations or repetitions based on the prior direction choice, or in the case of the first junction, based on the direction of the forced turn. This resulted in three binary data points per subject. There are eight different possibilities for an outcome of a subject $(000,001,010,100,011,101,110,111 ; 1=$ Alternation, $0=$ Repetition). The frequencies of those eight outcomes were counted and the overall distribution of walking patterns is presented in Figure 3. Using a chi square goodness of fit test, this distribution was shown to be significantly different from what would be expected if the subjects had alternated at a chance level $\left(\chi^{2}=34.76, p<0.001\right)$. To estimate an overall alternation rate while incorporating the threefold contribution to the dataset by each subject, we fitted a logistic regression to the data, which included subjects as a random factor (model: Alternation 1+(1|Subject) ). Based on the formula of the logistic regression, the estimated intercept of this model is equal to $\log \left(\frac{P(\text { alternation })}{1-P(\text { alternation })}\right)$. By looking at the intercept's confidence interval, we tested whether the intercept is significantly different from zero, which corresponds to an alternation rate of $50 \%$ $\left(\log \left(\frac{0.5}{0.5}\right)=0\right)$. The logistic regression showed an overall estimated alternation rate of $72 \%$ (defined as $P(X \mid Y)$ ), which was shown to be significantly different from a $50 \%$ alternation rate $(95 \%$ confidence interval: 0.65 - 0.79). In order to examine effects of gender and the sequential position of the junction (first, second or third junction) we fitted a second logistic regression, which included gender and

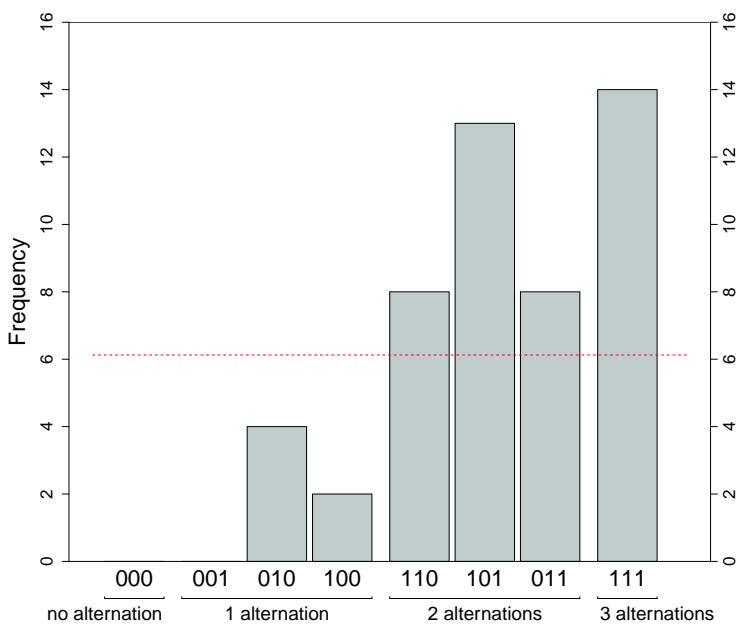

Figure 3: Distribution of the frequencies of the eigth possible maze trajectories ( $1=$ Alternation, $0=$ Repetition). The dashed red line indicates the frequencies expected by an alternation rate of $50 \%$.

junction position as fixed effects (model: Alternation $~ 1+$ Gender + JunctionPosition $+(1 \mid$ Subject $))$. The statistical significance of the effects gender and junction position were tested using likelihood ratio tests by comparing the full model to a reduced model missing the factor of interest respectively. The likelihood ratio tests however showed no significant effects of either gender nor junction position (gender: $\chi^{2}=0.02, p=0.88$; junction position: $\chi^{2}=0.20$, $p=0.65)$. To specifically test the alternation rate right after the primary forced turn regardless of direction (defined as $P(X \mid F Y)$ ) against a rate of $50 \%$, we conducted a binomial test. Results showed that subjects alternated with a rate of $76 \%$, which is significantly different from what would be expected by chance $(p<0.001$ (twosided)). Finally we also performed a binomial test on whether the alternation rate after two consecutive turns in the same direction (defined as $P(X \mid Y Y)$ ) is significantly higher than $50 \%$. The alternation rate following such two consecutive turns was $93 \%$, which is significantly different from what would be expected by chance $(\mathrm{p}<$ 0.001 (two-sided)). An overview of alternation rates can be found in Table 1. All tests were performed using the statistical software $\mathrm{R}$ [R Core Team 2017] [Bates et al. 2015] with a significance level of $\alpha=0.05$.

\section{DISCUSSION}

Our results indicate that in a maze-like VE, walking subjects alternate directions with a rate around $72 \%$. This alternation rate was shown to be independent of gender and sequential junction position, where the latter finding suggests that the alternation behavior does not decay or increase over time. Our finding seems to be comparable to alternation rates found when humans are navigating through a stylus maze as in [Lawless and Engstrand 1960] and surpasses the alternation rate so far found in walking humans [Neiberg et al. 1970]. Additionally we showed that a forced turn, in which subjects don't make a direction decision themselves, still resulted in an alternation rate of $76 \%$. This finding suggests that in the context of SAB it doesn't seem to make a difference for humans, 
Table 1: Summary of spontaneous alternation rates expressed as conditional probabilities given the prior turn direction(s). $P(R \mid F L)$ and $P(L \mid F R)$ are the alternation rates after the first forced left and right turn respectively. $P(X \mid F Y)$ is the alternation rate right after the primary forced turn regardless of direction. $P(X \mid Y)$ and $P(X \mid Y Y)$ are the overall estimated alternation rates after 1 and 2 turns in the same direction respectively. Number of subjects is indicated in brackets. (Stars indicate significant differences to $p=0.5$, $\alpha=0.05)$

\begin{tabular}{llllll}
\hline & $P(R \mid F L)$ & $P(L \mid F R)$ & $P(X \mid F Y)$ & $P(X \mid Y)$ & $P(X \mid Y Y)$ \\
\hline Male & 0.82 & 0.64 & $0.73^{*}$ & $0.73^{*}$ & $0.93^{*}$ \\
$(\mathrm{~N}=22)$ & $(11)$ & $(11)$ & $(22)$ & $(22)$ & $(14)$ \\
\hline Female & 0.75 & $0.80^{*}$ & $0.78^{*}$ & $0.72^{*}$ & $0.92^{*}$ \\
$(\mathrm{~N}=27)$ & $(12)$ & $(15)$ & $(27)$ & $(27)$ & $(13)$ \\
\hline Total & $0.78^{*}$ & $0.73^{*}$ & $0.76^{*}$ & $0.72^{*}$ & $0.93^{*}$ \\
$(\mathrm{~N}=49)$ & $(23)$ & $(26)$ & $(49)$ & $(49)$ & $(27)$ \\
\hline
\end{tabular}

whether a turn direction is self-chosen or imposed. When looking at the distribution of the eight possible maze trajectories in Figure 3 , a clear shift towards trajectories with two or three alternations can be observed, whereby all trajectories with one or no alternations occurred less than what would have been expected with a $50 \%$ alternation rate. Finally, when looking at junctions after two consecutive left or right turns, subjects changed direction with an even higher rate of $93 \%$. In general, the positive finding of alternation behavior in walking humans shows potential that this can be used to increase the efficiency of redirected walking using the MPC framework. However, how much of an enhancement the inclusion of an $\mathrm{SAB}$ rate would bring is still not clear and requires experimental testing. Moreover, while the alternation behavior seemed stable in our setting, it remains to be investigated how SAB can change in slightly different contexts. It was our effort to exclude other factors that might possibly influence alternation behavior as much as possible for this study: We didn't give the subjects any specific task, for example finding a way out of the maze; we created all junctions completely equal in terms of visual appearances including brightness. When considering more realistic VEs that might come up in real applications, these factors are not suppressed as they were in this experiment. Another point that should be addressed is that the distances between T-junctions in our maze were uniform and relatively short $(1.5 \mathrm{~m})$. In previous studies with humans, the distance between junctions has been shown to significantly influence alternation rates; longer distances between turns lead to lower alternation rates [Lawless and Engstrand 1960]. Finally, we mostly presented a first level alternation analysis, which assumes that alternation is only dependent on the immediately previous turn. A more in-depth analysis might be required to understand whether earlier turns also have an effect on the current turning choice.

\section{CONCLUSION}

In this paper, we described an experiment to investigate $\mathrm{SAB}$ in human adults during walking in a virtual maze. The alternation rate obtained could be used to update existing prediction models, thus enhancing the efficiency of the MPC planner. However, how much performance improvement could be achieved is not yet known, and will require further implementation and benchmarking. It would also be interesting to see if the behavior is affected when RDW techniques are applied. More specifically curvature gain, which forces the user onto a curved path through the insertion of a small rotation of the virtual scene, might influence the alternation rate in maze-like scenarios. Finally, the experiment is focused on testing $\mathrm{SAB}$ in a relative simple setting, without defined tasks, or other factors such as visual information (brightness, moving objects, etc.) and auditory information. The presence of these factors could significantly affect the alternation rate, and therefore requires further research.

\section{ACKNOWLEDGMENTS}

This work is funded by the Swiss National Foundation (SNF).

\section{REFERENCES}

Douglas Bates, Martin Mächler, Ben Bolker, and Steve Walker. 2015. Fitting Linear Mixed-Effects Models Using lme4. Journal of Statistical Software 67, 1 (2015), 1-48. https://doi.org/10.18637/jss.v067.i01

GA Dean. 1965. An analysis of the energy expenditure in level and grade walking. Ergonomics 8, 1 (1965), 31-47.

Timofey Grechkin, Jerald Thomas, Mahdi Azmandian, Mark Bolas, and Evan Suma. 2016. Revisiting detection thresholds for redirected walking: combining translation and curvature gains. In Proceedings of the ACM Symposium on Applied Perception. ACM, 113-120.

Lauren Harris. 1971. Variability in maze drawings of young children: Effects of stimulus change and chronological age. Psychonomic Science 23, 4 (1971), 305-307.

Richard Lawless and Raymond Engstrand. 1960. Alternation in the human stylus maze: Time and distance factors. 10 (1960), 101-105

Frank D Miller, Jane Dee-Wang Tu, Gene H Moffat, and Sharon Manley. 1969. Children's response alternation as a function of stimulus duration, age, and trials. Psychonomic Science 15, 4 (1969), 199-200.

Alan Neiberg, Jean Dale, and Darlene Grainger. 1970. Alternation of stimulus and response in three species. Psychonomic Science 18, 3 (1970), 183-184.

Thomas Nescher, Ying-Yin Huang, and Andreas Kunz. 2014. Planning redirection techniques for optimal free walking experience using model predictive control. In $3 D$ User Interfaces (3DUI), 2014 IEEE Symposium on. IEEE, 111-118.

James L Pate and Gail L Bell. 1971. Alternation behavior of children in a cross-maze. Psychonomic Science 23, 6 (1971), 431-432.

R Core Team. 2017. R: A Language and Environment for Statistical Computing. $\mathrm{R}$ Foundation for Statistical Computing, Vienna, Austria. https://www.R-project.org/

Sharif Razzaque, Zachariah Kohn, and Mary C Whitton. 2001. Redirected walking. In Proceedings of EUROGRAPHICS, Vol. 9. Citeseer, 105-106.

Frank Steinicke, Gerd Bruder, Jason Jerald, Harald Frenz, and Markus Lappe. 2008. Analyses of human sensitivity to redirected walking. In Proceedings of the 2008 ACM symposium on Virtual reality software and technology. ACM, 149-156.

Khrystyna Vasylevska, Hannes Kaufmann, Mark Bolas, and Evan A Suma. 2013. Flexible spaces: Dynamic layout generation for infinite walking in virtual environments. In 3D User Interfaces (3DUI), 2013 IEEE Symposium on. IEEE, 39-42.

Betsy Williams, Gayathri Narasimham, Bjoern Rump, Timothy P McNamara, Thomas H Carr, John Rieser, and Bobby Bodenheimer. 2007. Exploring large virtual environments with an HMD when physical space is limited. In Proceedings of the 4th symposium on Applied perception in graphics and visualization. ACM, 41-48. 